\title{
VARIANTS OF MIYACHI'S THEOREM FOR NILPOTENT LIE GROUPS
}

\author{
ALI BAKLOUTI and SUNDARAM THANGAVELU ${ }^{凶}$
}

(Received 27 February 2009; accepted 30 August 2009)

Communicated by A. M. Hassell

\begin{abstract}
We formulate and prove two versions of Miyachi's theorem for connected, simply connected nilpotent Lie groups. This allows us to prove the sharpness of the constant 1/4 in the theorems of Hardy and of Cowling and Price for any nilpotent Lie group. These theorems are proved using a variant of Miyachi's theorem for the group Fourier transform.
\end{abstract}

2000 Mathematics subject classification: primary 22E25; secondary 22G15.

Keywords and phrases: uncertainty principles, Plancherel formula, nilpotent Lie group.

\section{Introduction}

A theorem of Hardy [8], proved in 1933, says that a function $f$ and its Fourier transform $\hat{f}$ cannot both have arbitrarily rapid Gaussian decay unless $f$ is identically zero. Defining $\varphi_{a}(x)=e^{-a x^{2}}$, we can state Hardy's theorem more precisely as follows. If both $f / \varphi_{a}$ and $\hat{f} / \varphi_{b}$ are in $L^{\infty}(\mathbb{R})$ for some positive numbers $a$ and $b$, then $f=0$ whenever $a b>1 / 4$. Moreover, when $a b=1 / 4$ the function $f$ is a constant multiple of $\varphi_{a}$ and when $a b<1 / 4$ there are infinitely many linearly independent functions satisfying both conditions. In 1982, Cowling and Price [6] generalized Hardy's theorem by replacing the $L^{\infty}$ estimates by $L^{p}$ estimates. They proved that if $f / \varphi_{a} \in L^{p}(\mathbb{R})$ and $\hat{f} / \varphi_{b} \in L^{q}(\mathbb{R})$ for some $p$ and $q$ satisfying $1 \leq p, q \leq+\infty$, then $f=0$ whenever $a b>1 / 4$. The same conclusion holds even when $a b=1 / 4$ provided that at least one of $p$ and $q$ is finite. In 1997, Miyachi [10] proved the following generalization of Hardy's theorem.

THEOREM 1.1. Let $f$ be an integrable function on $\mathbb{R}$ such that

$$
f / \varphi_{a} \in L^{1}(\mathbb{R})+L^{\infty}(\mathbb{R}),
$$

The first author was supported by D.G.R.S.R.T., Research Unity 00 UR 1501 and the second author by a J.C. Bose Fellowship from DST.

(C) 2010 Australian Mathematical Publishing Association Inc. 1446-7887/2010 \$16.00 
for some positive a. Further assume that

$$
\int_{\mathbb{R}} \log ^{+}\left(\frac{e^{b \lambda^{2}}|\hat{f}(\lambda)|}{c}\right) d \lambda<+\infty,
$$

for some positive numbers $b$ and $c$. If $a b=1 / 4$, then $f$ is a constant multiple of the Gaussian $\varphi_{a}$.

Observe that when $f / \varphi_{a} \in L^{p}(\mathbb{R})$, it also belongs to $L^{1}(\mathbb{R})+L^{\infty}(\mathbb{R})$. The condition $\hat{f} / \varphi_{b} \in L^{q}(\mathbb{R})$ also implies that the integral

$$
\int_{-\infty}^{+\infty} \log ^{+}\left(\frac{e^{b \lambda^{2}}|\hat{f}(\lambda)|}{c}\right) d \lambda
$$

converges for some positive constant $c$. Thus Miyachi's theorem generalizes both the Hardy and the Cowling-Price theorems.

Over the last decade, the theorems of Hardy and of Cowling and Price have received considerable attention. Various generalizations of these theorems in several contexts such as semisimple Lie groups, symmetric spaces and nilpotent Lie groups have been proved. We refer to the surveys of Folland and Sitaram [7], Thangavelu [14], the papers [1-4] and the monograph [13] for more about such theorems. The first aim of this paper is to prove certain analogues of Miyachi's theorem in the context of nilpotent Lie groups. Recall that Kaniuth and Kumar [9] produced an analogue of Hardy's theorem for connected, simply connected nilpotent Lie groups. More precisely, they proved the following result.

THEOREM 1.2. Let $G$ be a connected, simply connected nilpotent Lie group. Let $f: G \rightarrow \mathbb{C}$ be a measurable function such that

$$
|f(x)| \leq C e^{-a\|x\|^{2}} \quad \forall x \in G
$$

for some positive a. Further assume that for some positive $b$,

$$
\left\|\pi_{\xi}(f)\right\|_{H S} \leq C e^{-b\|\xi\|^{2}}
$$

for all $\xi \in \mathcal{W}$, a suitable cross-section for the generic coadjoint orbits in $\mathfrak{g}^{*}$. Then $f$ is zero almost everywhere on $G$ whenever $a b>1 / 4$.

Precise definitions of the norms $x \mapsto\|x\|$ on $G$ and $\xi \mapsto\|\xi\|$ on $\mathfrak{g}^{*}$ and of the crosssection $\mathcal{W}$ will be given later. Recently, the first author of this paper and Ben Salah (see [3]) proved an analogue of the Cowling-Price theorem in the same context; they proved the following result.

THEOREM 1.3. Let $G$ be a connected, simply connected nilpotent Lie group and $f$ be a measurable function on $G$. Suppose that $2 \leq p, q \leq+\infty$, and let $a$ and $b$ be positive 
numbers such that

$$
\begin{gathered}
\int_{G} e^{p a\|x\|^{2}}|f(x)|^{p} d x<+\infty, \\
\int_{\mathcal{W}} e^{q b\|\xi\|^{2}}\left\|\pi_{\xi}(f)\right\|_{H S}^{q}|\operatorname{Pf}(\xi)| d \xi<+\infty,
\end{gathered}
$$

where $\operatorname{Pf}(\xi)$ stands for the Pfaffian at $\xi$. Then $f$ is zero almost everywhere on $G$ whenever $a b>1 / 4$.

The second aim of the present paper is to investigate the sharpness of the constant $1 / 4$ in both the Hardy and the Cowling-Price theorems. We will prove that the constant $1 / 4$ is sharp in Hardy's theorem for any nilpotent Lie group, provided that the second decay condition is replaced by

$$
|\operatorname{Pf}(\xi)|^{1 / 2}\left\|\pi_{\xi}(f)\right\|_{H S} \leq C e^{-b\|\xi\|^{2}} \quad \forall \xi \in \mathcal{W} .
$$

Such a result is currently known only for the Heisenberg group (see [13]). Some recent results concerning this point are also available for some restrictive classes of solvable Lie groups (see [2]). Concerning the Cowling-Price theorem, we will show that any function which fulfills both the conditions (1.5) and (1.6) of Theorem 1.3 above vanishes almost everywhere whenever $a b \geq 1 / 4$, provided that $\min (p, q)<+\infty$ and the second decay condition is replaced by

$$
\int_{\mathcal{W}} e^{q b\|\xi\|^{2}}\left\|\pi_{\xi}(f)\right\|_{H S}^{q}|\operatorname{Pf}(\xi)|^{q / 2} d \xi<+\infty .
$$

These results are immediate consequences of a variant of Miyachi's theorem. Our proofs make use of the orbit method and Plancherel theory. At present we do not know how to handle the case where $a b<1 / 4$ in the context of arbitrary nilpotent Lie groups. In the case of Heisenberg groups, we formulate and prove a heat kernel version of Miyachi's theorem. The main theorems are stated in the next section.

\section{Main results}

2.1. A variant of Miyachi's theorem for the real line. Note that when $a b>1 / 4$, Miyachi's theorem allows us to conclude that $f=0$. For applications to nilpotent Lie groups the following version (which is slightly weaker than the original result) is required. As we will see later, this is due to the fact that the function $x \mapsto \log ^{+} x$ is not convex whereas $x \mapsto x \log ^{+} x$ is. We first prove the following variant of Miyachi's theorem.

THEOREM 2.1. Let $f$ be an integrable function on $\mathbb{R}$ which satisfies

$$
\begin{gathered}
f / \varphi_{a} \in L^{1}(\mathbb{R})+L^{\infty}(\mathbb{R}), \\
\int_{\mathbb{R}} e^{b \lambda^{2}}|\hat{f}(\lambda)| \log ^{+}\left(\frac{e^{b \lambda^{2}}|\hat{f}(\lambda)|}{c}\right) d \lambda<+\infty
\end{gathered}
$$


for some positive numbers $a, b$ and $c$. If $a b>1 / 4$, then $f$ is zero almost everywhere. If $a b=1 / 4$, then $f$ is a constant multiple of the Gaussian $\varphi_{a}$. If $a b<1 / 4$, then there exist infinitely many linearly independent functions satisfying (2.1) and (2.2).

PROOF. First let us assume that $a b<1 / 4$. Then for any $r$ satisfying $a<r<1 / 4 b$, the Gaussian $\varphi_{r}$ meets the conditions (2.1) and (2.2). The case where $a b=1 / 4$ is a direct consequence of Miyachi's theorem. Now let $b^{\prime}$ be such that $a b \geq a b^{\prime}=1 / 4$. Then

$$
\begin{aligned}
\int_{\mathbb{R}} \log ^{+}\left(\frac{e^{b^{\prime} \lambda^{2}}|\hat{f}(\lambda)|}{c}\right) d \lambda & \leq c^{-1} \int_{\mathbb{R}} e^{b^{\prime} \lambda^{2}}|\hat{f}(\lambda)| \log ^{+}\left(\frac{e^{b^{\prime} \lambda^{2}}|\hat{f}(\lambda)|}{c}\right) d \lambda \\
& \leq c^{-1} \int_{\mathbb{R}} e^{b \lambda^{2}}|\hat{f}(\lambda)| \log ^{+}\left(\frac{e^{b \lambda^{2}}|\hat{f}(\lambda)|}{c}\right) d \lambda \\
& <+\infty .
\end{aligned}
$$

Thanks to Miyachi's theorem, we get $f=A \varphi_{a}$ and then $\hat{f}=B \varphi_{b^{\prime}}$ for some constants $A$ and $B$. This simply means that the integral (2.2) diverges unless $A=0$, as was to be shown.

COROLlARY 2.2. When hypothesis (2.2) is replaced by

$$
\int_{\mathbb{R}} e^{2 b \lambda^{2}}|\hat{f}(\lambda)|^{2} \log ^{+}\left(e^{b \lambda^{2}}|\hat{f}(\lambda)|\right) d \lambda<+\infty
$$

the same conclusions as in Theorem 2.1 hold.

2.2. Applications to nilpotent Lie groups. We assume henceforth that $G=\exp (\mathfrak{g})$ is a connected, simply connected nilpotent Lie group. For such groups, the exponential map is a global $C^{\infty}$ diffeomorphism and the unitary dual is homeomorphic to the space of coadjoint orbits when these spaces are endowed with their usual topologies. We begin by setting up notation in order to state our main results. Let

$$
\{0\}=\mathfrak{g}_{0} \subset \mathfrak{g}_{1} \subset \cdots \subset \mathfrak{g}_{n}=\mathfrak{g}
$$

be a Jordan-Hölder sequence for the nilpotent Lie algebra $\mathfrak{g}$, that is, a flag of ideals of $\mathfrak{g}$ such that $\operatorname{dim} \mathfrak{g}_{j}=j$, when $j=0, \ldots, n$. We extract from this sequence a Jordan-Hölder basis $\left\{X_{1}, \ldots, X_{n}\right\}$ by taking $X_{j} \in \mathfrak{g}_{j} \backslash \mathfrak{g}_{j-1}$, when $j=1, \ldots, n$. So obviously $\mathfrak{g}_{1}$, that is, $\mathbb{R} X_{1}$, is a central ideal of $\mathfrak{g}$. Let $\left\{X_{1}^{*}, \ldots, X_{n}^{*}\right\}$ be the basis of $\mathfrak{g}^{*}$ dual to $\left\{X_{1}, \ldots, X_{n}\right\}$; it is a Jordan-Hölder basis for the coadjoint action of $G$ on $\mathfrak{g}^{*}$. Given $\xi \in \mathfrak{g}^{*}$, an index $j \in\{1, \ldots, n\}$ is said to be a jump index for $\xi$ if

$$
\mathfrak{g}(\xi)+\mathfrak{g}_{j} \neq \mathfrak{g}(\xi)+\mathfrak{g}_{j-1}
$$

where $\mathfrak{g}(\xi)$ is the radical of the bilinear form $(X, Y) \mapsto B_{\xi}(X, Y)=\xi([X, Y])$. Let $e(\xi)$ be the set of jump indices for $\xi$. This set contains $\operatorname{dim}\left(O_{\xi}\right)$ indices, which is an even number. Moreover, there are disjoint sets of indices $S$ and $T$ such that $S \cup T=$ $\{1, \ldots, n\}$, and a $G$-invariant nonempty Zariski open set $\mathcal{U}$ of $\mathfrak{g}^{*}$ such that $e(\xi)=S$ for 
all $\xi \in \mathcal{U}$. We let $M_{S}(\xi)=\left(\xi\left(\left[X_{i}, X_{j}\right]\right)\right)_{i, j \in S}$, and then the Pfaffian $\operatorname{Pf}(\xi)$ is given by $|\operatorname{Pf}(\xi)|^{2}=\operatorname{det} M_{S}(\xi)$ for all $\xi$. Define $V_{T}=\operatorname{span}\left\{X_{i}^{*} \mid i \in T\right\}$, define $V_{S}$ analogously, and let $d \xi$ be the element of Lebesgue measure on $V_{T}$ normalized so that the unit cube spanned by $\left\{X_{i}^{*} \mid i \in T\right\}$ has volume 1 . Then $\mathfrak{g}^{*}=V_{T} \oplus V_{S}$ and $\mathcal{W}=\mathcal{U} \cap V_{T}$ is a cross-section for the coadjoint orbits through points in $\mathcal{U}$. Furthermore, if $d \xi$ also denotes the element of Lebesgue measure on $\mathcal{W}$, then $\mu$ is the Plancherel measure for $\hat{G}$, where $d \mu=|\operatorname{Pf}(\xi)| d \xi$. The Plancherel formula reads:

$$
\|\varphi\|_{2}^{2}=\int_{G}|\varphi(g)|^{2} d g=\int_{\mathcal{W}}\left\|\pi_{\xi}(\varphi)\right\|_{H S}^{2} d \mu(\xi) \quad \forall \varphi \in L^{1}(G) \cap L^{2}(G),
$$

where $\|T\|_{H S}$ denotes the Hilbert-Schmidt norm of an operator $T$ on a Hilbert space.

We now introduce a norm function on $G$ by setting

$$
\left\|\exp \left(x_{1} X_{1}+\cdots+x_{n} X_{n}\right)\right\|=\sqrt{x_{1}^{2}+\cdots+x_{n}^{2}} \quad \forall\left(x_{1}, \ldots, x_{n}\right) \in \mathbb{R}^{n} .
$$

The composed map from $\mathbb{R}^{n}$ via $\mathfrak{g}$ to $G$

$$
\left(x_{1}, \ldots, x_{n}\right) \mapsto \sum_{j=1}^{n} x_{j} X_{j} \mapsto \exp \left(\sum_{j=1}^{n} x_{j} X_{j}\right)
$$

is a diffeomorphism and maps the Lebesgue measure on $\mathbb{R}^{n}$ to the Haar measure on $G$. In this setup, we shall always identify $\mathfrak{g}$ and sometimes $G$ as sets with $\mathbb{R}^{n}$. We shall also identify $\mathfrak{g}^{*}$ with $\mathbb{R}^{n}$ by the map $\xi=\left(\xi_{1}, \ldots, \xi_{n}\right) \mapsto \sum_{j=1}^{n} \xi_{j} X_{j}^{*}$. We consider the Euclidean norm of $\mathfrak{g}^{*}$ with respect to the dual Jordan-Hölder basis $\left\{X_{1}^{*}, \ldots, X_{n}^{*}\right\}$, that is,

$$
\left\|\sum_{j=1}^{n} \xi_{j} X_{j}^{*}\right\|=\sqrt{\xi_{1}^{2}+\cdots+\xi_{n}^{2}}=\|\xi\| .
$$

Our first main result is the following theorem.

THEOREM 2.3. Let $G$ be a connected, simply connected nilpotent Lie group and $f$ be a measurable function on $G$ satisfying

$$
\begin{gathered}
e^{a\|\cdot\|^{2}} f \in L^{2}(G)+L^{\infty}(G), \\
\int_{\mathcal{W}} e^{b\|\xi\|^{2}}\left\|\pi_{\xi}(f)\right\|_{H S}^{2} \log ^{+}\left(\frac{e^{b\|\xi\|^{2}}\left\|\pi_{\xi}(f)\right\|_{H S}}{c}\right)|\operatorname{Pf}(\xi)| d \xi<+\infty
\end{gathered}
$$

for some positive numbers $a, b$ and $c$. Then $f$ is zero almost everywhere on $G$ whenever $a b>1 / 4$.

Using this result, we can obtain analogues of the Hardy and the Cowling-Price theorems for nilpotent Lie groups when $2 \leq p, q \leq+\infty$ proved in [3, 9].

Our second main result is concerned with another version of Miyachi's theorem. The idea is to seek the sharpness of the constant $1 / 4$ in Theorem 2.3 above, by mildly 
changing the second decay condition. Recall the Jordan-Hölder basis $\left\{X_{1}, \ldots, X_{n}\right\}$ of $\mathfrak{g}$ that was constructed through the ascending central series of $\mathfrak{g}$. So $\mathfrak{a}=\mathbb{R} X_{1}$ is contained in the center of $\mathfrak{g}$. For $y=\left(y_{2}, \ldots, y_{n}\right) \in \mathbb{R}^{n-1}$, define the complex valued function $f_{y}$ on $\mathbb{R}$ by

$$
f_{y}(t)=f(t, y)=f\left(\exp \left(t X_{1}+\sum_{j=2}^{n} y_{j} X_{j}\right)\right)=f\left(\exp t X_{1} \exp \left(\sum_{j=2}^{n} y_{j} X_{j}\right)\right)
$$

and $f_{y}^{*}(t)=\overline{f_{y}(-t)}$. We prove the following theorem.

THEOREM 2.4. Let $G$ be a connected, simply connected nilpotent Lie group and $f$ be a measurable function on $G$ satisfying

$$
\begin{aligned}
& e^{a\|\cdot\|^{2}} f \in L^{2}(G)+L^{\infty}(G), \\
& \int_{\mathcal{W}} e^{2 b\|\xi\|^{2}}\left\|\pi_{\xi}(f)\right\|_{H S}^{2} \log ^{+}\left(\frac{e^{b\|\xi\|^{2}}|\operatorname{Pf}(\xi)|^{1 / 2}\|\pi \xi(f)\|_{H S}}{c}\right) \\
& \quad \times|\operatorname{Pf}(\xi)| d \xi<+\infty
\end{aligned}
$$

for some positive numbers $a, b$ and $c$. If $a b>1 / 4$, then $f$ is zero almost everywhere on $G$ and if $a b=1 / 4$, then $f(t, y)=f(0, y) e^{-a t^{2}}$ for all $t \in \mathbb{R}$.

We now come back to Hardy's uncertainty principle. The following result is a direct consequence of Theorem 2.4 that shows the sharpness of the constant $1 / 4$ in the condition $a b=1 / 4$.

COROLlary 2.5. Let $G$ be a connected, simply connected nilpotent Lie group and $f: G \rightarrow \mathbb{C}$ be a measurable function such that for some positive numbers $a, b$ and $c$,

$$
\begin{gathered}
|f(x)| \leq c e^{-a \pi\|x\|^{2}} \quad \forall x \in G, \\
|\operatorname{Pf}(\xi)|^{1 / 2}\left\|\pi_{\xi}(f)\right\|_{H S} \leq c e^{-b \pi\|\xi\|^{2}} \quad \forall \xi \in \mathcal{W} .
\end{gathered}
$$

If $a b>1 / 4$, then $f$ is zero almost everywhere on $G$, and if $a b=1 / 4$, then $f(t, y)=$ $f(0, y) e^{-a t^{2}}$ for all $t \in \mathbb{R}$ and $y \in \mathbb{R}^{n-1}$.

Concerning the Cowling-Price theorem, we obtain the following generalization.

Corollary 2.6. Suppose that $G$ is a connected, simply connected nilpotent Lie group and that $p$ and $q$ satisfy $2 \leq p, q \leq+\infty$ and $\min (p, q)<+\infty$. Let $f$ be $a$ measurable function on $G$ such that, for some positive numbers $a$ and $b$,

$$
\begin{gathered}
\int_{G} e^{p a\|x\|^{2}}|f(x)|^{p} d x<+\infty \\
\int_{\mathcal{W}} e^{q b\|\xi\|^{2}}\left\|\pi_{\xi}(f)\right\|_{H S}^{q}|\operatorname{Pf}(\xi)|^{q / 2} d \xi<+\infty .
\end{gathered}
$$

Then $f$ is zero almost everywhere on $G$ whenever $a b \geq 1 / 4$. 
We now specialize to the case of the Heisenberg group $\mathbb{H}^{n}$. As a set, this is just $\mathbb{C}^{n} \times \mathbb{R}$. The case of Hardy's theorem where $a b=1 / 4$ for the Euclidean Fourier transform can be viewed as a result characterizing the heat kernel associated with the standard Laplacian on $\mathbb{R}^{n}$. Therefore it is natural to measure the decay of functions on the Heisenberg group $\mathbb{H}^{n}$ in terms of the heat kernel $q_{a}$ associated with the subLaplacian. Such a heat kernel version of Hardy's theorem for the Heisenberg group was proved by the second author in [12]. The Fourier transform $\hat{q}_{a}(\lambda)$ of the heat kernel is the operator $e^{-a H(\lambda)}$, where $H(\lambda)=-\Delta+\lambda^{2}|x|^{2}$ is the scaled Hermite operator on $\mathbb{R}^{n}$. Thus the Hardy condition on the Fourier transform side reads $\left\|\hat{f}(\lambda) e^{a H(\lambda)}\right\|_{\text {op }} \leq C$. Generalizing Hardy's theorem, a version of the Cowling-Price theorem was obtained in [11]. Following similar ideas, we can prove the following version of Miyachi's theorem for the Heisenberg group.

THEOREM 2.7. Suppose $f$ is a measurable function on $\mathbb{H}^{n}$ that satisfies

$$
\begin{gathered}
f / q_{a} \in L^{1}\left(\mathbb{H}^{n}\right)+L^{\infty}\left(\mathbb{H}^{n}\right), \\
\left\|\hat{f}(\lambda) e^{b H(\lambda)}\right\|_{\text {op }} \leq C \quad \forall \lambda \in \mathbb{R} \backslash\{0\} .
\end{gathered}
$$

Then $f$ is zero almost everywhere on $\mathbb{H}^{n}$ whenever $a<b$.

The proof of this theorem is very similar to that of Hardy's theorem and hence not given here. We refer to [13, Theorem 2.9.2] for details. As in Hardy's theorem, the equality case (where $a b=1 / 4$ ) remains open. In [13] the equality case was treated by replacing the assumption on $f$ by $\left|f^{\lambda}(z)\right| \leq C_{\lambda} q_{a}^{\lambda}(z)$ where $f^{\lambda}$ stands for the partial Fourier transform of $f$ in the central variable at $\lambda$. But unfortunately, we can only conclude that $f=q_{a} *_{3} \varphi$ where $\varphi$ is a tempered distribution on $\mathbb{R}$ with an $L^{\infty}$ Fourier transform. In the above, $*_{3}$ stands for convolution in the central variable. We therefore look for suitable conditions on $f$ and $\hat{f}$ which will completely characterize the heat kernel $q_{a}$.

Let us consider the Euclidean case for a moment, with $p_{a}^{n}$ standing for the heat kernel on $\mathbb{R}^{n}$. The conditions $\left|f * p_{b}^{n}(x)\right| \leq C p_{a+b}^{n}(x)$ and $|\hat{f}(\xi)| \leq C e^{-a|\xi|^{2}}$ imply that $f=c p_{a}^{n}$. Note that the heat kernel $p_{a}^{n}$ extends to $\mathbb{C}^{n}$ as an entire function and $p_{a}^{n}(i x)$ makes sense. Considering functions on $\mathbb{R}^{n+m}$, define $\tilde{p}_{a}^{n+m}(x, y)=$ $p_{a}^{n+m}(i x, y)$. Then $|f(x, y)| \leq C p_{a}^{n+m}(x, y)$ leads to $\left|f *_{m} \tilde{p}_{a}^{n+m}(x, y)\right| \leq C p_{2 a}^{m}(y)$ (here $*_{m}$ stands for convolution in the $y$ variable). It is easy to see that this latter condition on $f$ together with $|\hat{f}(\xi, \eta)| \leq C e^{-a\left(|\xi|^{2}+|\eta|^{2}\right)}$ leads to the conclusion that $f=c p_{a}^{n+m}$. Indeed, we let

$$
g_{\eta}(x)=\int_{\mathbb{R}^{n}} e^{-i y \cdot \eta} f(x, y) d y
$$

and then the condition on $f$ shows that $\left|g_{\eta}(x)\right| \leq C_{\eta} e^{-|x|^{2} / 4 a}$. This, together with the assumption on $\hat{f}$, allows us to conclude that $g_{\eta}=C(\eta) p_{a}^{n}$ or $f(x, y)=p_{a}^{n}(x) h(y)$ for all $x \in \mathbb{R}^{n}$ and $y \in \mathbb{R}^{m}$. Another application of Hardy's theorem proves that $h=C p_{a}^{m}$. 
With the above as a motivation we might try to holomorphically extend the heat kernel $q_{a}$ and use its restriction $q_{a}(i x, i y, i t)$ to measure the decay of the function $f$. Unfortunately, $q_{a}$ does not extend as an entire function and $q_{a}(i x, i y, i t)$ does not make sense for all $(x, y, t)$. Instead, we look at the heat kernel $k_{a}$ associated with the full Laplacian on $\mathbb{H}^{n}$ which is given by

$$
k_{a}(x, y, t)=\int_{-\infty}^{\infty} e^{-i \lambda t} e^{-a \lambda^{2}} q_{a}^{\lambda}(x, y) d \lambda
$$

Note that $k_{a}$ extends to $\mathbb{C}^{2 n+1}$ as an entire function. Rather than using $k_{a}(i x, i y, i t)$ we use the kernel

$$
\tilde{q_{a}}(x, y, t)=\int_{-\infty}^{\infty} e^{-i \lambda t} e^{-a \lambda^{2}}\left(\frac{\sinh \lambda a}{\lambda}\right)^{2 n} q_{a}^{\lambda}(i x, i y) d \lambda
$$

which is related to $k_{a}(i x, i y, t)$. Note that if $f=q_{a}$, then $f *_{3} \tilde{q_{a}}(x, y, t)=c e^{-t^{2} / 4 a}$ and $\hat{f}(\lambda) e^{a H(\lambda)}$ is a bounded operator for all $\lambda \neq 0$. These two properties characterize the heat kernel, as we show below.

THEOREM 2.8. Let $f$ be a measurable function on $\mathbb{H}^{n}$ that satisfies

$$
\begin{array}{cl}
\left|f *_{3} \tilde{q_{a}}(x, y, t)\right| \leq C e^{-t^{2} / 4 a} & \forall(x, y, t) \in \mathbb{H}^{n}, \\
\left\|\hat{f}(\lambda) e^{a H(\lambda)}\right\|_{\mathrm{op}} \leq C & \forall \lambda \neq 0 .
\end{array}
$$

Then $f$ is a constant multiple of $q_{a}$.

This is not yet the ideal analogue of Hardy's theorem. We would like to know whether the condition $|f(x, y, t)| \leq C q_{a}(x, y, t)$ for all $(x, y, t) \in \mathbb{H}^{n}$ implies assumption (2.9) of the theorem. It will be so if $\tilde{q_{a}}$ is nonnegative. Unfortunately, we do not know whether this is so or not. In the absence of this information, the theorem above is the best analogue of Hardy's theorem that we can prove. We also have the following version of Miyachi's theorem.

THEOREM 2.9. Let $f$ be a measurable function on $\mathbb{H}^{n}$ that satisfies

$$
f *_{3} \tilde{q_{a}}(x, y, t)=\left(f_{1}(x, y, t)+f_{\infty}(x, y, t)\right) e^{-t^{2} / 4 a},
$$

where $f_{1} \in L^{1}\left(\mathbb{H}^{n}\right)$ and $f_{\infty} \in L^{\infty}\left(\mathbb{H}^{n}\right)$, such that $\left\|f_{1}(x, y, \cdot)\right\|_{1} \leq C$ for all $x, y \in \mathbb{R}^{n}$ and

$$
\int_{\mathbb{R}} \log ^{+}\left(\frac{\left\|\hat{f}(\lambda) e^{a H(\lambda)}\right\|_{\mathrm{op}}}{c}\right) d \lambda<+\infty .
$$

Then $f$ is a constant multiple of $q_{a}$.

We prove this result in Section 3. Hardy's theorem follows as an immediate corollary. 


\section{Proofs of the main results}

We proceed in this section to prove our main results. We fix as above a JordanHölder basis $\left\{X_{1}, \ldots, X_{n}\right\}$ of $\mathfrak{g}$ such that $X_{1} \in \mathfrak{z}(\mathfrak{g})$ (the center of $\mathfrak{g}$ ), and define the function $f_{y}$ on $\mathbb{R}$ by $f_{y}(t)=f(t, y)$ for all $t \in \mathbb{R}$ and $y \in \mathbb{R}^{n-1}$.

Proof of Theorem 2.3. Define the function $g$ by

$$
g(t)=\int_{\mathbb{R}^{n-1}} f_{y} * f_{y}^{*}(t) d y .
$$

We prove our theorem by using some estimates on $g$ and its Euclidean Fourier transform $\hat{g}$. We begin with the following lemma on $g$.

LEMMA 3.1. Let $f$ satisfy the hypothesis of the theorem and $g$ be defined as above. Then for any $r$ satisfying $0<r<a / 2$,

$$
g / \varphi_{r} \in L^{1}(\mathbb{R})+L^{\infty}(\mathbb{R}) .
$$

In particular, $g$ is integrable with respect to Lebesgue measure.

PROOF. Since $e^{a\|\cdot\|^{2}} f \in L^{2}(G)+L^{\infty}(G)$, we have $e^{a\|\cdot\|^{2}} f=u_{1}+u_{\infty}$ where $u_{\infty} \in$ $L^{\infty}(G)$ and $u_{1}$ is in $L^{2}(G)$ and vanishes outside a set of finite measure, hence is also in $L^{1}(G)$. Then the function $f$ defined by $F(x)=f(x)-e^{-a\|x\|^{2}} u_{1}(x)$ satisfies $|F(x)| \leq M e^{-a\|x\|^{2}}$. From [9], therefore, $|\tilde{F}(t)| \leq M e^{-a t^{2} / 2}$ for all $t \in \mathbb{R}$, where $\tilde{F}$ is the function defined on $\mathbb{R}$ by

$$
\tilde{F}(t)=\int_{\mathbb{R}^{n-1}} F_{y} * F_{y}^{*}(t) d y .
$$

We then have

$$
\tilde{F}(t)=\int_{\mathbb{R}^{n-1}}\left(\left\{f_{y}-e^{-a|\cdot|^{2}} e^{-a\|y\|^{2}}\left(u_{1}\right)_{y}\right\} *\left\{f_{y}^{*}-e^{-a|\cdot|^{2}} e^{-a\|y\|^{2}}\left(u_{1}\right)_{y}^{*}\right\}\right)(t) d y .
$$

Thus we see that $\tilde{F}(t)=J_{1}(t)-J_{2}(t)-J_{3}(t)+J_{4}(t)$, where $J_{1}(t)=g(t)$,

$$
\begin{aligned}
& J_{2}(t)=\int_{\mathbb{R}^{n-1}}\left(f_{y} *\left\{e^{-a|\cdot|^{2}} e^{-a\|y\|^{2}}\left(u_{1}\right)_{y}^{*}\right\}\right)(t) d t, \\
& J_{3}(t)=\int_{\mathbb{R}^{n-1}}\left(\left\{e^{-a|\cdot|^{2}} e^{-a\|y\|^{2}}\left(u_{1}\right)_{y}\right\} * f_{y}^{*}\right)(t) d t
\end{aligned}
$$

and

$$
J_{4}(t)=\int_{\mathbb{R}^{n-1}}\left(\left\{e^{-a|\cdot|^{2}} e^{-a\|y\|^{2}}\left(u_{1}\right)_{y}\right\} *\left\{e^{-a|\cdot|^{2}} e^{-a\|y\|^{2}}\left(u_{1}\right)_{y}^{*}\right\}\right)(t) d y .
$$

Thus $\tilde{F}(t)=g(t)+h(t)$ where the function $h$ is defined as a sum of the last three integrals, each of which is of the form $\int_{\mathbb{R}^{n-1}} U_{y} * V_{y}(t) d y$ for some functions $U$ and $V$ 
on the group $G$. Thus $e^{a t^{2} / 2} g(t)=e^{a t^{2} / 2} \tilde{F}(t)-e^{a t^{2} / 2} h(t)$. As $e^{a t^{2} / 2}|\tilde{F}(t)| \leq C$ for all $t \in \mathbb{R}$, it is sufficient to show that $h / \varphi_{a / 2} \in L^{1}(\mathbb{R})$. If $r<a / 2$, then

$$
\begin{aligned}
\int_{\mathbb{R}} e^{r t^{2}}\left|J_{2}(t)\right| d t & \leq \int_{\mathbb{R}} \int_{\mathbb{R}^{n-1}} \int_{\mathbb{R}}\left|f_{y}(s)\right| e^{-a(t-s)^{2}} e^{-a\|y\|^{2}} e^{r t^{2}}\left|u_{1}(s-t, y)\right| d t d y d s \\
& =\int_{\mathbb{R}} \int_{\mathbb{R}^{n-1}}\left|f_{y}(s)\right| \int_{\mathbb{R}} e^{-a u^{2}} e^{-a\|y\|^{2}} e^{r(s-u)^{2}}\left|u_{1}(u, y)\right| d u d y d s \\
& \leq \int_{\mathbb{R}} \int_{\mathbb{R}^{n-1}}\left|f_{y}(s)\right| \int_{\mathbb{R}} e^{-a u^{2}} e^{-a\|y\|^{2}} e^{2 r\left(s^{2}+u^{2}\right)}\left|u_{1}(u, y)\right| d u d y d s \\
& =\int_{\mathbb{R}} \int_{\mathbb{R}^{n-1}}\left|f_{y}(s)\right| e^{2 r s^{2}} \int_{\mathbb{R}} e^{(2 r-a) u^{2}} e^{-a\|y\|^{2}}\left|u_{1}(u, y)\right| d u d y d s \\
& =\int_{\mathbb{R}^{n-1}}\left(\int_{\mathbb{R}}\left|f_{y}(s)\right| e^{2 r s^{2}} d s\right)\left(\int_{\mathbb{R}} e^{(2 r-a) u^{2}} e^{-a\|y\|^{2}}\left|u_{1}(u, y)\right| d u\right) d y
\end{aligned}
$$

which is dominated by

$$
\begin{aligned}
& \left(\int_{\mathbb{R}^{n-1}}\left\{\int_{\mathbb{R}}\left|f_{y}(s)\right| e^{2 r s^{2}} d s\right\}^{2} d y\right)^{1 / 2} \\
& \quad \times\left(\int_{\mathbb{R}^{n-1}}\left\{\int_{\mathbb{R}} e^{-(a-2 r) u^{2}} e^{-a\|y\|^{2}}\left|u_{1}(u, y)\right| d u\right\}^{2} d y\right)^{1 / 2} .
\end{aligned}
$$

As the integral

$$
\int_{\mathbb{R}^{n-1}}\left(\int_{\mathbb{R}} e^{-(a-2 r) u^{2}} e^{-a\|y\|^{2}}\left|u_{1}(u, y)\right| d u\right)^{2} d y
$$

converges (by the Cauchy-Schwarz inequality), we see that

$$
\int_{\mathbb{R}} e^{r t^{2}}\left|J_{2}(t)\right| d t \leq M\left(\int_{\mathbb{R}^{n-1}}\left(\int_{\mathbb{R}}\left|f_{y}(s)\right| e^{2 r s^{2}} d s\right)^{2} d y\right)^{1 / 2},
$$

for some positive $M$. Choose a positive $\varepsilon$ such that $2 r+2 \varepsilon=a_{0}<a$. We then have

$$
\int_{\mathbb{R}} e^{r t^{2}}\left|J_{2}(t)\right| d t \leq M\left(\int_{\mathbb{R}^{n-1}}\left(\int_{\mathbb{R}} e^{-2 \varepsilon s^{2}}\left|f_{y}(s)\right| e^{a_{0} s^{2}} d s\right)^{2} d y\right)^{1 / 2} .
$$

With $a_{1}=a-a_{0}$, the last integral is dominated by

$$
\int_{\mathbb{R}^{n-1}}\left(\int_{\mathbb{R}} e^{-\varepsilon s^{2}} e^{-\varepsilon s^{2}}\left|f_{y}(s)\right| e^{a\left(s^{2}+\|y\|^{2}\right)} e^{-a_{1}\left(s^{2}+\|y\|^{2}\right)} d s\right)^{2} d y .
$$

Recalling the definition of $u_{1}$ and $u_{\infty}$, the above integral is bounded by

$$
\int_{\mathbb{R}^{n-1}}\left(\int_{\mathbb{R}} e^{-\varepsilon s^{2}} e^{-\varepsilon s^{2}}\left(\left|u_{1}(s, y)\right|+\left|u_{\infty}(s, y)\right|\right) d s\right)^{2} e^{-2 a_{1}\|y\|^{2}} d y .
$$


Therefore $\int_{\mathbb{R}} e^{r t^{2}}\left|J_{2}(t)\right| d t$ is bounded by a constant multiple of

$$
\int_{\mathbb{R}^{n-1}} \int_{\mathbb{R}} e^{-2 \varepsilon s^{2}} e^{-2 a_{1}\|y\|^{2}}\left(\left|u_{1}(s, y)\right|^{2}+\left|u_{\infty}(s, y)\right|^{2}+2\left|u_{1}(s, y)\right|\left|u_{\infty}(s, y)\right|\right) d s d y,
$$

which is finite. This completes the estimate of $J_{2}$. The same computation can be done for the remaining integrals defining the function $h$. We first pay attention to the function $J_{3}$. Using the computations above, we get

$$
\int_{\mathbb{R}} e^{r t^{2}}\left|J_{3}(t)\right| d t \leq \int_{\mathbb{R}} \int_{\mathbb{R}^{n-1}} \int_{\mathbb{R}}\left|f_{y}(-s)\right| e^{-a(t-s)^{2}} e^{-a\|y\|^{2}} e^{r t^{2}}\left|u_{1}(t-s, y)\right| d t d y d s
$$

which, after a change of variables, can be put in the form

$$
\int_{\mathbb{R}} \int_{\mathbb{R}^{n-1}}\left|f_{y}(s)\right| \int_{\mathbb{R}} e^{-a u^{2}} e^{-a\|y\|^{2}} e^{r(s+u)^{2}}\left|u_{1}(u, y)\right| d u d y d s
$$

and the estimate for $J_{3}$ follows. We finally tackle the integral $\int_{\mathbb{R}} e^{r t^{2}}\left|J_{4}(t)\right| d t$. Denote by $H$ the function on $G$ such that $H_{y}(t)=e^{-a|t|^{2}} e^{-a\|y\|^{2}} u_{1}(t, y)$ for all $y \in \mathbb{R}^{n-1}$; then we easily see that $x \mapsto e^{a\|x\|^{2}}|H(x)| \in L^{1}(G)$ and the computations above allow us to show that this integral converges.

We now consider the Fourier transform $\hat{g}$ of the function $g \in L^{1}(\mathbb{R})$. An easy computation shows that

$$
\hat{g}(\xi)=\int_{\mathbb{R}^{n-1}}\left|\hat{f}_{y}(\xi)\right|^{2} d y
$$

and consequently $\hat{g} \geq 0$ and

$$
\int_{\mathbb{R}} \hat{g}(\xi) d \xi=\int_{\mathbb{R}^{n}}\left|f_{y}(\xi)\right|^{2} d y d \xi=\|f\|_{2}^{2} .
$$

In view of (3.2), the function $f$ is zero almost everywhere on $G$ if and only if the function $g$ is zero almost everywhere on $\mathbb{R}$.

We need a localized version of the Plancherel measure (see [5]). Let $Z(G)$ be the (closed connected) center of $G$ and $A$ be a closed connected subgroup of $Z(G)$ with Lie algebra $\mathfrak{a}$. Then $Z(G)=\exp \mathfrak{z}(\mathfrak{g})$ and $A=\exp \mathfrak{a}$. For $\psi \in \mathfrak{a}^{*}$, define the (unitary) character $\chi_{\psi}$ of $A$ by the formula

$$
\chi_{\psi}\left(\exp \left(s X_{1}\right)\right)=e^{-2 \pi i s \xi_{1}} .
$$

Take $\chi=\chi_{\psi}$, and let

$$
\hat{G}_{\chi}=\left\{\pi \in \hat{G}: \pi_{\mid A}=\chi \cdot I\right\} .
$$

Then $\hat{G}_{\chi}$ is a closed subset of $\hat{G}$ and is homeomorphic to the orbit space $\mathfrak{g}_{\psi}^{*} / G$, where $\mathfrak{g}_{\psi}^{*}=\psi+\mathfrak{a}^{\perp}$. Furthermore, it is the unitary dual (the set of unitary and topologically irreducible representations) of the convolution algebra $L^{1}(G / A, \psi)$. Here, $L^{p}(G / A, \psi)$ is defined (when $1 \leq p<+\infty$ ) as the set of all measurable 
functions $\varphi: G \rightarrow \mathbb{C}$ such that $\varphi(g a)=\overline{\chi(a)} \varphi(g)$ for all $g \in G$ and $a \in A$, and

$$
\|\varphi\|_{L^{p}(G / A, \psi)}^{p}=\int_{G / A}|\varphi(g)|^{p} d g<+\infty .
$$

Note that $L^{1}(G / A, \psi)$ is a Banach *-algebra. The convolution of $\varphi$ and $\varphi^{\prime}$ in $L^{1}(G / A, \psi)$ is defined here by

$$
\varphi * \varphi^{\prime}(g)=\int_{G / A} \varphi(u) \varphi^{\prime}\left(u^{-1} g\right) d u \quad \forall g \in G
$$

and the involution is defined by $f^{*}(x)=\overline{f\left(x^{-1}\right)}$. It is easy to check that the function $\varphi * \varphi^{\prime}$ has the required covariance relation with respect to the character $\chi$. So in this case, the localized Plancherel formula reads: for $\varphi \in L^{2}(G / A, \psi)$, if

$$
\tilde{\pi}(\varphi)=\int_{G / A} \varphi(g) \tilde{\pi}(g) d g \quad \forall \tilde{\pi} \in \hat{G}_{\chi},
$$

then

$$
\int_{G / A}|\varphi(g)|^{2} d g=\int_{\hat{G}_{\chi}} \operatorname{tr} \tilde{\pi}\left(\varphi^{*} * \varphi\right) d \mu_{\chi}(\tilde{\pi})
$$

where the measure $\mu_{\chi}$ is obtained in the following way. Let $\mathcal{W}_{\psi}=\mathcal{W} \cap \mathfrak{g}_{\psi}^{*}$. For $\varphi \in L^{1}(G / A, \psi) \cap L^{2}(G / A, \psi)$,

$$
\|\varphi\|_{2}=\left(\int_{\mathcal{W}_{\psi}}\left\|\pi_{l}(\varphi)\right\|_{H S}^{2}|\operatorname{Pf}(l)| d l\right)^{1 / 2} .
$$

We henceforth take $\mathfrak{a}=\mathbb{R} X_{1}$. If $d$ is the maximal dimension of coadjoint orbits in $\mathfrak{g}^{*}$, then $T$ has $n-d$ elements and so $V_{T}$ can be identified with $\mathbb{R}^{n-d}$. In an abuse of notation we write $V_{T}=\mathbb{R} X_{1}^{*} \oplus \mathbb{R}^{n-d-1}$ and let

$$
p^{*}: V_{T} \rightarrow \mathbb{R} X_{1}^{*}, \quad \xi \mapsto \xi_{1} X_{1}^{*}
$$

denote the canonical projection. As $\mathcal{W}$ is a Zariski open set of $V_{T}, p^{*}(\mathcal{W})=\mathcal{O}$ is also a nonempty Zariski open set of $\mathbb{R}$. So it will be convenient to write elements $\xi \in \mathcal{W}$ as $\left(\xi_{1}, \xi^{\prime}\right)$, where $\xi_{1} \in \mathcal{O}$ and $\xi^{\prime} \in \mathcal{W}_{\xi_{1}}=\left\{\xi^{\prime} \in \mathbb{R}^{n-d-1} \mid\left(\xi_{1}, \xi^{\prime}\right) \in \mathcal{W}\right\}$. It turns out that $\mathcal{W}_{\xi_{1}}$ is also a Zariski open set of $\mathbb{R}^{n-d-1}$ for each fixed $\xi_{1} \in \mathcal{O}$. The set $\mathcal{W}_{\xi_{1}}$ corresponds obviously to the cross-section $\mathcal{W}_{\psi}$ used in the localized version of the Plancherel formula in (3.3). We now prove the following lemma.

LEMMA 3.2. For all $\xi_{1} \in \mathcal{O}$,

$$
\hat{g}\left(\xi_{1}\right)=\int_{\mathcal{W}_{\xi_{1}}}|\operatorname{Pf}(\xi)|\left\|\pi_{\xi}(f)\right\|_{H S}^{2} d \xi^{\prime} .
$$

PRoOF. Let $\xi_{1} \in \mathcal{O}$, and $V_{n}\left(\xi_{1}\right)=\left(\xi_{1}-1 / 2 n, \xi_{1}+1 / 2 n\right)$ for all $n \in \mathbb{N}^{*}$. From [9, p. 491],

$$
\hat{g}\left(\xi_{1}\right)=\lim _{n \rightarrow+\infty} n \int_{V_{n}\left(\xi_{1}\right)} \int_{\mathcal{W}_{\eta_{1}}}|\operatorname{Pf}(\eta)|\left\|\pi_{\eta}(f)\right\|_{H S}^{2} d \eta^{\prime} d \eta_{1},
$$


where $\eta=\left(\eta_{1}, \eta^{\prime}\right)$. Now $\pi_{\eta}(f)=\tilde{\pi}_{\eta}\left(f_{\eta_{1}}\right)$, where $\tilde{\pi}_{\eta}=\pi_{\chi_{\eta_{1}}}$ and $f_{\eta_{1}}\left(g_{1}\right)=\mathcal{F}^{1} f\left(\eta_{1}, g_{1}\right)$. It follows that

$$
\hat{g}\left(\xi_{1}\right)=\lim _{n \rightarrow+\infty} \int_{G / A} n \int_{V_{n}\left(\xi_{1}\right)}\left|f_{\eta_{1}}\left(g_{1}\right)\right|^{2} d \eta_{1} d g_{1} .
$$

We will use the dominated convergence theorem. We first observe that

$$
n \int_{V_{n}\left(\xi_{1}\right)}\left|f_{\eta_{1}}\left(g_{1}\right)\right|^{2} d \eta_{1}=\left|f_{c_{n}}\left(g_{1}\right)\right|^{2}
$$

for some $c_{n} \in V_{n}\left(\xi_{1}\right)$. Moreover,

$$
\left|f_{c_{n}}\left(g_{1}\right)\right|^{2}=\left|\int_{\mathbb{R}} f\left(g_{1} e^{s X_{1}}\right) e^{-2 \pi i s c_{n}} d s\right|^{2} \leq\left(\int_{\mathbb{R}}\left|f\left(g_{1} e^{s X_{1}}\right)\right| d s\right)^{2} .
$$

If we can show that the integral

$$
E=\int_{G / A}\left(\int_{\mathbb{R}}\left|f\left(g_{1} e^{s X_{1}}\right)\right| d s\right)^{2} d g_{1}
$$

converges, then we can apply the dominated convergence theorem. But this is easy to see. We first identify $G / A$ with $\mathbb{R}^{n-1}$ by a Borel cross-section. Let $r$ be a positive number such that $4 r<a$. Then

$$
\begin{aligned}
E & \leq \int_{\mathbb{R}^{n-1}}\left(\int_{\mathbb{R}} e^{-r s^{2}} e^{-r s^{2}} e^{-2 r\|y\|^{2}} e^{2 r\|(s, y)\|^{2}}|f(s, y)| d s\right)^{2} d y \\
& \leq \int_{\mathbb{R}^{n-1}} \int_{\mathbb{R}^{2}} e^{-2 r s^{2}} e^{-4 r\|y\|^{2}} e^{4 r\|(s, y)\|^{2}}|f(s, y)|^{2} d s d y \\
& \leq \int_{\mathbb{R}^{n}} e^{-2 r s^{2}} e^{-4 r\|y\|^{2}}\left(\left|u_{1}(s, y)\right|+\left|u_{\infty}(s, y)\right|\right)^{2} d s d y \\
& <+\infty
\end{aligned}
$$

which proves our claim. Here $u_{1}$ and $u_{\infty}$ are as in the proof of Lemma 3.1.

Finally, we can apply the dominated convergence theorem to get

$$
\begin{aligned}
\hat{g}\left(\xi_{1}\right) & =\int_{G / A} \lim _{n \rightarrow+\infty}\left|f_{c_{n}}\left(g_{1}\right)\right|^{2} d g_{1} \\
& =\int_{G / A}\left|f_{\xi_{1}}\left(g_{1}\right)\right|^{2} d g_{1} \\
& =\int_{\mathcal{W}_{\xi_{1}}}\left\|\pi_{\eta}(f)\right\|_{H S}^{2}|\operatorname{Pf}(\eta)| d \eta^{\prime},
\end{aligned}
$$

using (3.3) again. This completes the proof of the lemma.

We can now complete the proof of Theorem 2.3 by showing that $g=0$. Suppose that $\varepsilon<b, 2 r<a$ and $2 \varepsilon r>1 / 4$. Writing $\eta=\left(\lambda, \eta^{\prime}\right)$,

$$
e^{2 \varepsilon \lambda^{2}} \hat{g}(\lambda) \leq \int_{\mathcal{W}_{\lambda}} e^{2 \varepsilon\|\eta\|^{2}}\left\|\pi_{\eta}(f)\right\|_{H S}^{2}|\operatorname{Pf}(\eta)| d \eta^{\prime} .
$$


Let $t(\lambda)=\int_{\mathcal{W}_{\lambda}} e^{-2 \varepsilon\|\eta\|^{2}}|\operatorname{Pf}(\eta)| d \eta^{\prime}$, for all $\lambda \in \mathbb{R}^{*}$. Then clearly $t$ is a bounded continuous function on $\mathbb{R}$ that never vanishes on $\mathbb{R}^{*}$. Let $C=\|t\|_{\infty}$, and consider

$$
\begin{aligned}
\frac{e^{2 \varepsilon \lambda^{2}} \hat{g}(\lambda)}{C c^{2}} & =\int_{\mathcal{W}_{\lambda}} \frac{e^{4 \varepsilon\|\eta\|^{2}}\left\|\pi_{\eta}(f)\right\|_{H S}^{2}}{C c^{2}} e^{-2 \varepsilon\|\eta\|^{2}}|\operatorname{Pf}(\eta)| d \eta^{\prime} \\
& =\int_{\mathcal{W}_{\lambda}}\left[\frac{t(\lambda) e^{4 \varepsilon\|\eta\|^{2}}\left\|\pi_{\eta}(f)\right\|_{H S}^{2}}{C c^{2}}\right] \frac{e^{-2 \varepsilon\|\eta\|^{2}}|\operatorname{Pf}(\eta)|}{t(\lambda)} d \eta^{\prime} .
\end{aligned}
$$

Let

$$
K(\lambda)=e^{2 \varepsilon \lambda^{2}} \hat{g}(\lambda) \log ^{+} \frac{e^{2 \varepsilon \lambda^{2}} \hat{g}(\lambda)}{C c^{2}} .
$$

Applying Jensen's inequality to the convex function $x \mapsto x \log ^{+} x$ and the probability measure with element $\left(e^{-2 \varepsilon\|\cdot\|^{2}}|\operatorname{Pf}(\cdot)|\right) /(t(\lambda)) d \eta^{\prime}$, we see that

$$
\begin{aligned}
K(\lambda) & \leq \int_{\mathcal{W}_{\lambda}} e^{2 \varepsilon\|\eta\|^{2}}\left\|\pi_{\eta}(f)\right\|_{H S}^{2} \log ^{+}\left(\frac{t(\lambda) e^{2 \varepsilon\|\eta\|^{2}}\left\|\pi_{\eta}(f)\right\|_{H S}^{2}}{C c^{2}}\right)|\operatorname{Pf}(\eta)| d \eta^{\prime} \\
& \leq 2 \int_{\mathcal{W}_{\lambda}} e^{2 \varepsilon\|\eta\|^{2}}\left\|\pi_{\eta}(f)\right\|_{H S}^{2} \log ^{+}\left(\frac{e^{\varepsilon\|\eta\|^{2}}\left\|\pi_{\eta}(f)\right\|_{H S}}{c}\right)|\operatorname{Pf}(\eta)| d \eta^{\prime} \\
& \leq 2 \int_{\mathcal{W}_{\lambda}} e^{b\|\eta\|^{2}}\left\|\pi_{\eta}(f)\right\|_{H S}^{2} \log ^{+}\left(\frac{e^{b\|\eta\|^{2}}\left\|\pi_{\eta}(f)\right\|_{H S}}{c}\right)|\operatorname{Pf}(\eta)| d \eta^{\prime} .
\end{aligned}
$$

We finally get that

$$
\begin{aligned}
\int_{\mathbb{R}} K(\lambda) d \lambda & \leq 2 \int_{\mathcal{W}} e^{b\|\eta\|^{2}}\left\|\pi_{\eta}(f)\right\|_{H S}^{2} \log ^{+}\left(\frac{e^{b\|\eta\|^{2}}\left\|\pi_{\eta}(f)\right\|_{H S}}{c}\right)|\operatorname{Pf}(\eta)| d \eta \\
& <+\infty
\end{aligned}
$$

As $2 r \varepsilon>1 / 4$, Theorem 2.1 shows that $g$ vanishes almost everywhere, proving the theorem.

Proof of Theorem 2.4. For a fixed Schwartz function $\varphi$ on $\mathbb{R}^{n-1}$, define the function $F$ on $\mathbb{R}$ by

$$
F(t)=\int_{\mathbb{R}^{n-1}} f(t, y) \varphi(y) d y \quad \forall t \in \mathbb{R} .
$$

Starting from the first decay condition of Theorem 2.4 concerning $f$, we observe that

$$
F(t)=\int_{\mathbb{R}^{n-1}} e^{-a\left(t^{2}+\|y\|^{2}\right)}\left(u_{1}(t, y)+u_{\infty}(t, y)\right) \varphi(y) d y
$$

for all $t \in \mathbb{R}$, and so

$$
e^{a t^{2}} F(t)=\int_{\mathbb{R}^{n-1}} e^{-a\|y\|^{2}} u_{1}(t, y) \varphi(y) d y+\int_{\mathbb{R}^{n-1}} e^{-a\|y\|^{2}} u_{\infty}(t, y) \varphi(y) d y .
$$


Let

$$
F_{1}(t)=\int_{\mathbb{R}^{n-1}} e^{-a\|y\|^{2}} u_{1}(t, y) \varphi(y) d y
$$

and

$$
F_{\infty}(t)=\int_{\mathbb{R}^{n-1}} e^{-a\|y\|^{2}} u_{\infty}(t, y) \varphi(y) d y
$$

Then it is clear that $F_{1} \in L^{1}(\mathbb{R})$ and $F_{\infty} \in L^{\infty}(\mathbb{R})$. On the other hand,

$$
\hat{F}(\lambda)=\int_{\mathbb{R}^{n-1}} \hat{f}_{y}(\lambda) \varphi(y) d y,
$$

and therefore

$$
|\hat{F}(\lambda)|^{2} \leq M \int_{\mathbb{R}^{n-1}}\left|\hat{f}_{y}(\lambda)\right|^{2} d y
$$

for some positive constant $M$. By (3.1), this implies that

$$
|\hat{F}(\lambda)|^{2} \leq M \hat{g}(\lambda)=M \int_{\mathcal{W}_{\lambda}}\left\|\pi_{\eta}(f)\right\|_{H S}^{2}|\operatorname{Pf}(\eta)| d \eta^{\prime},
$$

which leads to the estimate

$$
e^{2 b \lambda^{2}}|\hat{F}(\lambda)|^{2} \leq M C \int_{\mathcal{W}_{\lambda}} e^{2 b\|\eta\|^{2}}\left\|\pi_{\eta}(f)\right\|_{H S}^{2}|\operatorname{Pf}(\eta)| \frac{e^{-2 b\left\|\eta^{\prime}\right\|^{2}}}{C} d \eta^{\prime},
$$

where $C$ is chosen so that $\left(e^{-2 b\left\|\eta^{\prime}\right\|^{2}}\right) / C$ is the density of a probability measure on $\mathcal{W}_{\lambda}$. Using Jensen's inequality as before, we see that the expression

$$
\left(1 / M c^{2}\right) e^{2 b \lambda^{2}}|\hat{F}(\lambda)|^{2} \log ^{+}\left(\frac{e^{b \lambda^{2}}|\hat{F}(\lambda)|}{c \sqrt{M c}}\right)
$$

is dominated by

$$
\frac{1}{c^{2}} \int_{W_{\lambda}} e^{2 b\|\eta\|^{2}}\left\|\pi_{\eta}(f)\right\|_{H S}^{2} \log ^{+}\left(\frac{e^{b\|\eta\|^{2}}\left\|\pi_{\eta}(f)\right\|_{H S}|\operatorname{Pf}(\eta)|^{1 / 2}}{c}\right)|\operatorname{Pf}(\eta)| d \eta^{\prime} .
$$

This proves that the integral

$$
\int_{\mathbb{R}} e^{2 b \lambda^{2}}|\hat{F}(\lambda)|^{2} \log ^{+}\left(\frac{e^{b \lambda^{2}}|\hat{F}(\lambda)|}{c \sqrt{M c}}\right) d \lambda
$$

converges. We now make use of Corollary 2.2. When $a b>1 / 4$, we see that $f$ vanishes almost everywhere. When $a b=1 / 4$, a similar argument to that in [13] shows that $f(t, y)=f(0, y) e^{-a t^{2}}$ for all $t \in \mathbb{R}$ and $y \in \mathbb{R}^{n-1}$, as was to be shown. 
Proof of Corollary 2.6. The function $e^{a\|\cdot\|^{2}} f$ belongs to $L^{p}(G)$ by assumption, and hence to $L^{1}(G)+L^{\infty}(G)$. On the other hand, whenever $q>2$, the function $f$ fulfills the second decay condition of Theorem 2.4. This implies that $f$ vanishes almost everywhere whenever $a b>1 / 4$. In the case where $a b=1 / 4$, the function takes the form $f(t, y)=f(0, y) e^{-a t^{2}}$ for all $t \in \mathbb{R}$ and $y \in \mathbb{R}^{n-1}$. This implies that the integral

$$
\int_{G} e^{p a\|x\|^{2}}|f(x)|^{p} d x
$$

diverges unless $f(0, \cdot)$ vanishes almost everywhere on $\mathbb{R}^{n-1}$, as was to be shown.

We now look at the case where $q=2$. The fact that $e^{a\|\cdot\|^{2}} f$ belongs to $L^{p}(G)$ shows that $t \mapsto e^{a t^{2}} F(t)$ belongs to $L^{p}(\mathbb{R})$, where $F$ is the function defined in the proof of Theorem 2.3. Now

$$
\begin{aligned}
\int_{\mathbb{R}} e^{2 b \lambda^{2}}|\hat{F}(\lambda)|^{2} d \lambda & \leq \int_{\mathbb{R}} e^{2 b \lambda^{2}}\left(\int_{\mathbb{R}^{n-1}}|f(\lambda, y) \varphi(y)| d y\right)^{2} d \lambda \\
& =\int_{\mathbb{R}} e^{2 b\left(\lambda^{2}+|y|^{2}\right)}\left(\int_{\mathbb{R}^{n-1}} e^{-b|y|^{2}}|f(\lambda, y) \varphi(y)| d y\right)^{2} d \lambda \\
& \leq M \int_{\mathbb{R}} \int_{\mathbb{R}^{n-1}} e^{2 b\|(\lambda, y)\|^{2}}|f(\lambda, y)|^{2} d y d \lambda \\
& <+\infty,
\end{aligned}
$$

for some positive constant $M$. By the classical Cowling-Price theorem for the real line, $F$ is zero almost everywhere whenever $a b \geq 1 / 4$ and hence so is $f$.

PROOF OF THEOREM 2.8. By taking the inverse Fourier transform in the central variable, we observe that the first assumption on $f$ leads to

$$
\left|f^{\lambda}(x, y)\right| \tilde{q}_{a}^{\lambda}(x, y) \leq\left\|f_{1}(x, y, \cdot)\right\|_{1}+\|f\|_{\infty} \leq C .
$$

Recalling the definition of $\tilde{q_{a}}$ we observe that

$$
{\tilde{q_{a}}}^{\lambda}(x, y)=c_{n} e^{-a \lambda^{2}}\left(\frac{\sinh \lambda a}{\lambda}\right)^{2 n} q_{a}^{\lambda}(i x, i y)=c_{n} e^{-a \lambda^{2}} q_{a}^{\lambda}(x, y)^{-1} .
$$

Therefore the above condition on $f^{\lambda}$ can be rewritten as $\left|f^{\lambda}(x, y)\right| \leq C(\lambda) q_{a}^{\lambda}(x, y)$ for all $x, y \in \mathbb{R}^{n}$ and $\lambda \neq 0$. This, together with the second assumption, namely, $\left\|\hat{f}(\lambda) e^{a H(\lambda)}\right\|_{\text {op }} \leq C$, allows us to proceed as in [13, Theorem 2.9.6] to conclude that $f^{\lambda}(x, y)=C(\lambda) q_{a}^{\lambda}(x, y)$. The function $g(\cdot)=f *_{3} \tilde{q_{a}}(0,0, \cdot)$ satisfies $g(t)=$ $\left(g_{1}(t)+g_{\infty}(t)\right) e^{-t^{2} / 4 a}$ with $g_{1} \in L^{1}(\mathbb{R})$ and $g_{\infty} \in L^{\infty}(\mathbb{R})$. Moreover,

$$
\hat{g}(\lambda)=f^{\lambda}(0,0) q_{a}^{\lambda}(0,0)^{-1} e^{-a \lambda^{2}}=C(\lambda) e^{-a \lambda^{2}}
$$

so that

$$
\hat{g}(\lambda) e^{a \lambda^{2}}=C(\lambda)=\left\|\hat{f}(\lambda) e^{a H(\lambda)}\right\|_{\mathrm{op}}
$$


Therefore the function $g$ satisfies the hypotheses of Miyachi's theorem and hence $g(t)=c e^{-t^{2} / 4 a}$. This means that $C(\lambda)$ is a constant and hence $f^{\lambda}=c q_{a}^{\lambda}$ for all $\lambda$, or $f=c q_{a}$, proving the theorem.

\section{Acknowledgement}

The authors are immensely grateful to the referee for his extremely careful reading of the first version of this paper. His valuable suggestions were followed in revising the manuscript.

\section{References}

[1] A. Baklouti and E. Kaniuth, 'On Hardy's uncertainty principle for connected nilpotent Lie groups', Math. Z. 259(2) (2008), 233-247.

[2] A. Baklouti and E. Kaniuth, 'On Hardy's uncertainty principle for solvable locally compact groups', J. Fourier Anal. Appl., to appear.

[3] A. Baklouti and N. Ben Salah, 'The $L^{p}-L^{q}$ version of Hardy's theorem on nilpotent Lie groups', Forum Math. 18(2) (2006), 245-262.

[4] A. Baklouti and N. Ben Salah, 'On theorems of Beurling and Cowling-Price for certain nilpotent Lie groups', Bull. Sci. Math. 132(6) (2008), 529-550.

[5] A. Baklouti, K. Smaoui and J. Ludwig, 'Estimate of $L^{p}$-Fourier transform norm on nilpotent Lie groups', J. Funct. Anal. 199 (2003), 508-520.

[6] M. Cowling and J. Price, 'Generalizations of Heisenberg's inequality', in: Harmonic Analysis, Lecture Notes in Mathematics, 992 (eds. G. Mauceri, F. Ricci and G. Weiss) (Springer, Berlin, 1983).

[7] G. Folland and A. Sitaram, 'The uncertainty principle: a mathematical survey', J. Fourier Anal. Appl. 3(3) (1997), 207-238.

[8] G. H. Hardy, 'A theorem concerning Fourier transforms', J. London Math. Soc. 8 (1933), 227-231.

[9] E. Kaniuth and A. Kumar, 'Hardy's theorem for nilpotent Lie groups', Math. Proc. Cambridge Philos. Soc. 131 (2001), 487-494.

[10] A. Miyachi, 'A generalization of a theorem of Hardy', Harmonic Analysis Seminar held at Izunagaoka, Shizuoka-ken, Japan, (1997), 44-51.

[11] S. Parui and S. Thangavelu, 'Variations on a theorem of Cowling and Price with applications to nilpotent Lie groups', J. Aust. Math. Soc. 82 (2007), 11-27.

[12] S. Thangavelu, 'Hardy's theorem on the Heisenberg group revisited', Math. Z. 242 (2002), 761-779.

[13] S. Thangavelu, An Introduction to the Uncertainty Principle: Hardy's Theorem on Lie Groups, Progress in Mathematics, 217 (Birkhäuser, Boston, MA, 2004).

[14] S. Thangavelu, 'A survey of Hardy type theorems', in: Advances in Analysis, (eds. H. G. W. Begehr, R. P. Gilbert, M. E. Muldoon and M. W. Wong) (World Scientific, Singapore, 2005), pp. 39-70.

\section{ALI BAKLOUTI, Department of Mathematics, Faculty of Sciences at Sfax, Route de Soukra, 3038, Sfax, Tunisia \\ e-mail: Ali.Baklouti@fss.rnu.tn}

SUNDARAM THANGAVELU, Department of Mathematics, Indian Institute of Science, Bangalore 560012, India e-mail: veluma@math.iisc.ernet.in 